Accelerator Division

Alternating Gradient Synchrotron Department BROOKHAVEN NATIONAL LABORATORY

Upton, New York 11973

Accelerator Division

Technical Note

AGS/AD/Tech. Note No. 417

HEAVY ION PARAMETERS FOR 1995

C.J. Gardner

September 1, 1995 


\title{
Heavy Ion Parameters for 1995
}

\author{
C. J. Gardner
}

September 1, 1995

Ions of Iron and Gold will be accelerated in the Tandem, Booster, and AGS for the 1995 HIP (Heavy Ion Physics) run. During the first two weeks of the run, NASA will require Iron ions at $1 \mathrm{GeV}$ kinetic energy per nucleon to bombard various biological samples. These ions will be extracted by slow extraction from the AGS and transported to experimental area A3. For the remainder of the run, Gold ions will be delivered to the experimental areas and to the ATR (AGS To RHIC) line. The experiments require Slow Extracted Beam while the ATR line will require a single bunch kicked out of the AGS with the new Fast Extracted Beam setup. All experiments except one will require Gold ions at the same momentum they had last year, namely $11.6 \mathrm{GeV} / \mathrm{c}$ per nucleon. The other experiment will require Gold ions with kinetic energies of 2 to $4 \mathrm{GeV}$ per nucleon. Following is a summary of various ion parameters during injection, acceleration, and extraction in the Tandem, Booster and AGS.

\section{Charge States and Atomic Parameters}

Ions from the negative ion source enter the tandem with charge minus one, and mass and kinetic energy

$$
m_{s}=a u+m_{e}, \quad W_{s}=130 \mathrm{keV},
$$

where $a$ is the atomic mass, $u=931.49432 \mathrm{MeV} / c^{2}$ is the unified atomic mass unit, and $m_{e} c^{2}=.5110034 \mathrm{MeV}$ is the electron mass. The atomic masses of Iron and Gold are 55.847 and 196.966541 respectively. In the 
tandem, the negative ions are accelerated from ground potential to the center terminal where they are stripped of $1+Q_{t}$ electrons thereby becoming positive ions (with charge $Q_{t}$ ) which are then accelerated back to ground potential. The total energy gained by each ion as it passes through the tandem is then

$$
W_{t}=\left(1+Q_{t}\right) V_{t}
$$

where $V_{t}$ is the terminal voltage. A foil located downstream of the tandem and upstream of the first $90^{\circ}$ bend in the TTB (Tandem To Booster) line may be inserted to allow additional stripping of the ions before they enter the booster. The charge state after this stripping is $Q_{b}$. Ions with this charge are transported down the TTB line and injected into the booster where they undergo further acceleration. After extraction from the booster they pass through a foil in the BTA (Booster To AGS) line and emerge with charge $Q_{a}$. Ions with this charge are transported down the BTA line and injected into the AGS where they are again accelerated. After extraction from the AGS the ions are either transported to fixed-target experiments in the experimental hall or they are sent down the ATR line where they pass through a final foil which strips away the remaining electrons. The charge of the fully stripped ion is $Q_{r}$. The nominal charge states for the ions of the 1995 HIP run are summarized in the following table. (Note that the subscripts $t, b, a$, and $r$ are used to denote the charge states in the tandem, booster, ags, and rhic respectively. $n$ and $a$ are number of nucleons and atomic mass.)

\begin{tabular}{|c|c|c|c|c|c|c|}
\hline \multicolumn{6}{|c|}{ Table I: Charge States and Atomic Parameters } \\
\hline Atom & $n$ & $a$ & $Q_{t}$ & $Q_{b}$ & $Q_{a}$ & $Q_{r}$ \\
\hline Iron & 56 & 55.847 & +10 & +10 & +26 & +26 \\
Gold & 197 & 196.966541 & +12 & +32 & +77 & +79 \\
\hline
\end{tabular}

\section{Booster Injection}

The momentum of ions transported down the TTB line and injected into the booster is determined by the setting of the magnetic field (as measured by NMR probes) in the $90^{\circ}$ bends of the TTB line. The tandem voltage required to give the desired momentem is then determined by adjusting the 
voltage so that the ion beam is centered in slits located downstream of the bends. If we let $p$ be the momentum of the ions injected into the booster, then their energy is

$$
E=\sqrt{p^{2} c^{2}+m^{2} c^{4}}
$$

where the mass is

$$
m=a u-Q_{b} m_{e} .
$$

We define the kinetic energy to be

$$
W=E-m c^{2},
$$

and to a good approximation we then have

$$
W_{s}+W_{t}=W .
$$

The tandem voltage is therefore (approximately)

$$
V_{t}=W_{t} /\left(1+Q_{t}\right)=\left(W-W_{s}\right) /\left(1+Q_{t}\right) .
$$

It is convenient to parameterize the momentum and energy in terms of the kinetic energy defined by (5). Thus using (3) and (5) we have

$$
c p=\sqrt{W^{2}+2 m c^{2} W}, \quad E=m c^{2}+W .
$$

We can then derive all other injection parameters from $c p$ and $E$. Thus the rigidity of the ion beam in units of $\mathrm{Tm}$ is

$$
B \rho=k p / Q
$$

where $k=3.33564095 \times 10^{-3}, p$ is the momentum in units of $\mathrm{MeV} / \mathrm{c}$, and $Q$ is the ion charge. The inflector voltage, $V_{I}$, required to bring the beam into the acceptance region of the booster is

$$
e V_{I}=\frac{D}{R} c^{2} p^{2} /(Q E)
$$

where $D=0.017 \mathrm{~m}$ and $R=8.74123 \mathrm{~m}$ (see Booster Tech. Note No. 159, February 28, 1990). The velocity and revolution frequency of the ion as it enters the booster are

$$
v=c \beta=c^{2} p / E, \quad f=v / C, \quad C=2 \pi R_{a} / 4=\pi R_{a} / 2
$$


where $C$ is the booster circumference and $R_{a}=128.454$ meters is the radius of the AGS. The frequency of the accelerating voltage in the booster is $h f$, where $h=8$ for the 1995 run. The following table summarizes the various tandem and booster injection parameters. For the 1995 run, the nominal injection parameters for Iron and Gold are those corresponding to kinetic energies $W=127$ and $182.13 \mathrm{MeV}$ respectively.

\begin{tabular}{|c|c|c|c|c|c|c|}
\hline \multicolumn{7}{|c|}{ Table II: Tandem and Booster Injection Parameters } \\
\hline Ion & $W(\mathrm{MeV})$ & $V_{t}(\mathrm{MV})$ & $c p(\mathrm{MeV} / n)$ & $B \rho(\mathrm{Tm})$ & $V_{I}(\mathrm{kV})$ & $h f(\mathrm{kHz})$ \\
\hline \multirow{3}{*}{$\mathrm{Fe}^{10+}$} & 126.0 & 11.443 & 64.69099 & 1.20840 & 48.950 & 825.823 \\
& 127.0 & 11.534 & 64.94750 & 1.21319 & 49.338 & 829.081 \\
& 128.0 & 11.625 & 65.20301 & 1.21797 & 49.726 & 832.327 \\
\hline \multirow{3}{*}{$\mathrm{Au}^{32+}$} & 181.13 & 13.923 & 41.39216 & .849990 & 22.006 & 527.795 \\
& 182.13 & 14.000 & 41.50632 & .852334 & 22.127 & 529.247 \\
& 183.13 & 14.077 & 41.62017 & .854672 & 22.248 & 530.696 \\
\hline
\end{tabular}

\section{Booster Extraction}

For the 1995 run, the ions are accelerated in the booster at harmonic $h=8$ to $h f=5.0 \mathrm{MHz}$ (which is the upper limit of the booster RF system) and are then kicked out of the booster into the BTA line. Since the maximum energy to which a given ion can be accelerated is limited by the maximum field of the booster magnets, it is convenient to parameterize the booster extraction parameters in terms of the magnetic rigidity $B \rho$. Thus we have

$$
p=Q_{b} B \rho / k, \quad E=\sqrt{p^{2} c^{2}+m^{2} c^{4}}, \quad W=E-m c^{2}, \quad f=c^{2} p /(E C)
$$

where $m$ is given by (4). We also define $P$ to be the momentum of a proton which has the same rigidity as the ion under consideration. Thus

$$
P=B \rho / k=p / Q_{b} .
$$

The following table summarizes the various booster extraction parameters. 


\begin{tabular}{|c|c|c|c|c|c|}
\hline \multicolumn{7}{|c|}{ Table III: Booster Extraction Parameters } \\
\hline Ion & $B \rho(\mathrm{Tm})$ & $c P(\mathrm{GeV})$ & $c p(\mathrm{MeV} / n)$ & $W(\mathrm{MeV} / n)$ & $h f(\mathrm{MHz})$ \\
\hline \multirow{3}{*}{$\mathrm{Fe}^{10+}$} & 7.6451 & 2.292 & 409.2756 & 86.1709 & 4.792704 \\
& 8.0451 & 2.412 & 430.6893 & 94.9928 & 5.000007 \\
& 8.4451 & 2.532 & 452.1031 & 104.1833 & 5.201911 \\
\hline & 8.4670 & 2.538 & 412.3196 & 87.1966 & 4.812133 \\
$\mathrm{Au}^{32+}$ & 8.8670 & 2.658 & 431.7985 & 95.2372 & 4.999994 \\
& 9.2670 & 2.778 & 451.2774 & 103.5820 & 5.183411 \\
\hline
\end{tabular}

\section{$4 \quad$ AGS Injection}

Ions are injected into the AGS after having been stripped to charge $Q_{a}$ in the BTA line. It is convenient to parameterize the injection parameters in terms of the AGS revolution frequency, $f_{a}$. Thus we have

$$
v=2 \pi R_{a} f_{a}, \quad \beta=v / c, \quad \gamma=1 / \sqrt{1-\beta^{2}}
$$

and

$$
c p=m c^{2} \beta \gamma, \quad E=m c^{2} \gamma, \quad W=E-m c^{2}, \quad B \rho=k p / Q_{a}
$$

where $2 \pi R_{a}$ is the AGS circumference and

$$
m=a u-Q_{a} m_{e} .
$$

We also define $P=B \rho / k=p / Q_{a}$. The following table summarizes the various parameters. The harmonic number at injection is $h_{a}=12$.

\begin{tabular}{|c|c|c|c|c|c|}
\hline \multicolumn{7}{|c|}{ Table IV: AGS Injection Parameters } \\
\hline Ion & $h_{a} f_{a}(\mathrm{MHz})$ & $B \rho(\mathrm{Tm})$ & $c P(\mathrm{GeV})$ & $c p(\mathrm{MeV} / n)$ & $W(\mathrm{MeV} / n)$ \\
\hline \multirow{3}{*}{$\mathrm{Fe}^{26+}$} & 1.797264 & 2.9400 & 0.881 & 409.2113 & 86.1574 \\
& 1.875003 & 3.0938 & 0.927 & 430.6216 & 94.9779 \\
& 1.950717 & 3.2476 & 0.974 & 452.0320 & 104.1669 \\
\hline \multirow{3}{*}{$\mathrm{Au}^{77+}$} & 1.804550 & 3.5183 & 1.055 & 412.2680 & 87.1857 \\
& 1.874998 & 3.6845 & 1.105 & 431.7444 & 95.2252 \\
& 1.943779 & 3.8507 & 1.154 & 451.2209 & 103.5690 \\
\hline
\end{tabular}

Note that we have chosen the frequencies, $h_{a} f_{a}$, listed in Table IV so that $h\left(4 f_{a}\right)$ is equal to the corresponding frequency listed in the last column of 
Table III. In other words, we have chosen $f_{a}=f / 4$, which is the frequency one would expect if the ion velocity did not change between the booster and AGS. The ions do, of course, lose electrons and energy as they pass through the stripping foil and so the AGS revolution frequency, $f_{a}$, is in fact less than one fourth the booster revolution frequency $f$. The loss can actually be quite significant. During the 1994 heavy ion run, the loss in momentum for gold ions was found to be about $14 \mathrm{MeV} / \mathrm{c}$ per nucleon. The numbers listed in Table IV therefore serve only as a guide to the expected values of the AGS injection parameters.

\section{AGS Extraction}

As with the Booster, it is convenient to parameterize the AGS extraction parameters in terms of the magnetic rigidity, $B \rho$, or the corresponding proton momentum $P=B \rho / k$. Thus we have

$$
p=Q_{a} B \rho / k, \quad E=\sqrt{p^{2} c^{2}+m^{2} c^{4}}, W=E-m c^{2}, \quad f_{a}=c^{2} p /(4 E C)
$$

where $m$ is given by (16) and $4 C=2 \pi R_{a}$ is the AGS circumference. The following table summarizes the various parameters. For the 1995 run, $h_{a}=12$.

\begin{tabular}{|c|c|c|c|c|c|}
\hline \multicolumn{7}{|c|}{ Table V: AGS Extraction Parameters } \\
\hline Ion & $B \rho(\mathrm{Tm})$ & $c P(\mathrm{GeV})$ & $c p(\mathrm{GeV} / n)$ & $W(\mathrm{GeV} / n)$ & $h_{a} f_{a}(\mathrm{Mhz})$ \\
\hline & 10.8105 & 3.2409 & 1.50470 & 0.83952 & 3.793032 \\
$\mathrm{Fe}^{26+}$ & 12.1447 & 3.6409 & 1.69042 & 1.00002 & 3.906573 \\
& 13.4790 & 4.0409 & 1.87613 & 1.16470 & 3.994689 \\
\hline & 23.7187 & 7.1107 & 2.77931 & 2.00000 & 4.226442 \\
$\mathrm{Au}^{77+}$ & 32.5939 & 9.7714 & 3.81928 & 3.00001 & 4.330487 \\
& 41.3256 & 12.3891 & 4.84244 & 4.00001 & 4.377141 \\
\hline & 94.2472 & 28.2546 & 11.04368 & 10.15172 & 4.441567 \\
$\mathrm{Au}^{77+}$ & 95.5815 & 28.6546 & 11.20002 & 10.30752 & 4.442002 \\
& 96.9157 & 29.0546 & 11.35637 & 10.46334 & 4.442419 \\
\hline \multirow{3}{*}{$\mathrm{Au}^{77+}$} & $\mathbf{9 8 . 3 2 8 0}$ & 29.4780 & 11.52186 & 10.62829 & 4.442842 \\
& 98.9952 & 29.6780 & 11.60003 & 10.70621 & 4.443036 \\
& 99.6623 & 29.8780 & 11.67820 & 10.78413 & 4.443226 \\
\hline
\end{tabular}

